\title{
An international multicenter study of early intravenous fluid administration and outcome in acute pancreatitis
}

United European Gastroenterology Journal 2017, Vol. 5(4) 491-498 (C) Author(s) 2016 Reprints and permissions: sagepub.co.uk/journalsPermissions.nav DOI: $10.1177 / 2050640616671077$ journals.sagepub.com/home/ueg @SAGE

\author{
Vikesh K Singh ${ }^{1}$, Timothy B Gardner ${ }^{2}$, Georgios I Papachristou ${ }^{3}$, \\ Mónica Rey-Riveiro ${ }^{4}$, Mahya Faghih ${ }^{1}$, Efstratios Koutroumpakis ${ }^{3}$, \\ Elham Afghani ${ }^{1}$, Nelly G Acevedo-Piedra ${ }^{4}$, Nikhil Seth ${ }^{3}$, \\ Amitasha Sinha ${ }^{1}$, Noé Quesada-Vázquez ${ }^{4}$, Neftali Moya-Hoyo ${ }^{4}$, \\ Claudia Sánchez-Marin ${ }^{4}$, Juan Martínez ${ }^{4}$, Félix Lluís", \\ David C Whitcomb ${ }^{3}$, Pedro Zapater ${ }^{5}$ and Enrique de-Madaria ${ }^{4}$
}

\begin{abstract}
Aims: Early aggressive fluid resuscitation in acute pancreatitis is frequently recommended but its benefits remain unproven. The aim of this study was to determine the outcomes associated with early fluid volume administration in the emergency room (FVER) in patients with acute pancreatitis.

Methods: A four-center retrospective cohort study of 1010 patients with acute pancreatitis was conducted. FVER was defined as any fluid administered from the time of arrival to the emergency room to $4 \mathrm{~h}$ after diagnosis of acute pancreatitis, and was divided into tertiles: nonaggressive $(<500 \mathrm{ml})$, moderate $(500$ to $1000 \mathrm{ml})$, and aggressive $(>1000 \mathrm{ml})$.

Results: Two hundred sixty-nine (26.6\%), 427 (42.3\%), and 314 (31.1\%) patients received nonaggressive, moderate, and aggressive FVER respectively. Compared with the nonaggressive fluid group, the moderate group was associated with lower rates of local complications in univariable analysis, and interventions, both in univariable and multivariable analysis (adjusted odds ratio (95\% confidence interval): $0.37(0.14-0.98)$ ). The aggressive resuscitation group was associated with a significantly lower need for interventions, both in univariable and multivariable analysis (adjusted odds ratio 0.21 (0.05-0.84)). Increasing fluid administration categories were associated with decreasing hospital stay in univariable analysis. Conclusions: Early moderate to aggressive FVER was associated with lower need for invasive interventions.
\end{abstract}

Keywords

Resuscitation, treatment, prognosis, acute pancreatitis, outcome

Received: 24 July 2016; accepted: 29 August 2016

\section{Introduction}

Acute pancreatitis is the third most frequent cause of hospitalization for gastrointestinal disease in the USA. ${ }^{1}$ As there are no targeted pharmacologic therapies for acute pancreatitis, management is largely focused on supportive measures.

Consensus guidelines have advocated using aggressive fluid administration as a means of improving outcomes in acute pancreatitis since the late $1990 \mathrm{~s}^{2-7}$ Experimental studies in animal models as well as indirect and retrospective findings from clinical studies have suggested that better outcomes are achieved using aggressive fluid administration. ${ }^{8-15}$ These studies
${ }^{1}$ Pancreatitis Center, Division of Gastroenterology, Johns Hopkins Medical Institutions, Baltimore, USA

${ }^{2}$ Section of Gastroenterology and Hepatology, Dartmouth-Hitchcock Medical Center, Lebanon, USA

${ }^{3}$ Division of Gastroenterology and Hepatology, University of Pittsburgh Medical Center, Pittsburgh, USA

${ }^{4}$ Department of Gastroenterology, Hospital General Universitario de Alicante, Instituto de Investigación Sanitaria y Biomédica de Alicante (ISABIAL - Fundación FISABIO), Spain

${ }^{5}$ Clinical Pharmacology, Hospital General Universitario de Alicante, Instituto de Investigación Sanitaria y Biomédica de Alicante (ISABIAL-Fundación FISABIO), Spain

Corresponding author:

Enrique de-Madaria, Servicio de Aparato Digestivo, Hospital General Universitario de Alicante, C/Pintor Baeza s/n. 03010, Alicante, Spain. Email: madaria@hotmail.com 
hypothesized that aggressive fluid administration would improve perfusion of the pancreatic microcirculation, preventing pancreatic necrosis and attenuating the systemic inflammatory response syndrome (SIRS), which would avert organ failure and death..$^{3,16-19}$ On the other hand, several recent retrospective $e^{20,21}$ and prospective $^{22-24}$ studies have reported that the administration of high fluid volumes is associated with increased local complications, organ failure, and death. All of the studies addressing the relationship between the initial 24 to $72 \mathrm{~h}$ of fluid volume administration and outcomes in acute pancreatitis are potentially affected by reverse causation bias. Reverse causation bias refers to the scenario in which the outcome precedes and causes the exposure instead of the other way around. ${ }^{25}$ Patients with local complications and/or SIRS have increased fluid sequestration ${ }^{26}$ resulting in oliguria, hemoconcentration and hypotension. As a result, clinicians will administer higher volume of fluid to these patients, which is then blamed for any subsequent poor outcomes. ${ }^{27}$ Reverse causation bias is difficult to control for and may be the primary shortcoming of most studies that have evaluated fluid administration in acute pancreatitis, which have shown either benefit $^{9-14}$ or harm ${ }^{20,21,24}$ associated with aggressive fluid resuscitation.

There may also be a short "therapeutic window" during which aggressive fluid administration is useful for achieving positive outcomes ${ }^{7,24,28}$ without contributing to complications caused by overly aggressive resuscitation. Given the concerns surrounding reverse causation bias and timing of fluid administration, we hypothesized that examining the volume of fluid administration in the emergency room (ER) potentially addresses these concerns since fluid is administered early in the course of disease and is less influenced by subsequent fluid sequestration, so the primary aim of our study was to investigate the effect of early fluid volume administration in the emergency room (FVER) on outcomes of acute pancreatitis.

\section{Methods}

The relationship between fluid volume and outcome of acute pancreatitis was studied in separate cohorts of consecutive patients from four institutions which included the Hospital General Universitario de Alicante (HGUA), Alicante, Spain; the DartmouthHitchcock Medical Center (DHMC), Lebanon, New Hampshire, USA; the University of Pittsburgh Medical Center (UPMC), Pittsburgh, Pennsylvania, USA; and Johns Hopkins Medical Institutions (JHMI), Baltimore, Maryland, USA. Only adult ( $\geq 18$ years of age) patients with first or recurrent acute pancreatitis were included. Acute pancreatitis was defined according to the revised Atlanta classification. ${ }^{29}$ Prediction of severity was assessed using the Bedside Index of Severity in Acute Pancreatitis (BISAP) score determined at ER (by retrieving retrospectively the individual variables from the databases). ${ }^{30,31}$ Patients with chronic pancreatitis, with missing or incomplete data regarding fluid administration in the ER, those undergoing chronic hemodialysis, and those transferred from outside institutions were excluded from the analysis. Data were prospectively recorded in the HGUA cohort (Fluid Resuscitation database). Data from UPMC were prospectively recorded, with the exception of fluid administration, which was acquired retrospectively (review of medical records). Data from both DHMC and JHMI were retrospectively recorded (review of medical records). The study period included the index hospital admission, and further hospital admissions due to symptomatic local complications. The study was approved by the human research review committee of the involved centers.

\section{Explicative variables}

FVER was defined as any fluid administered from the time of arrival at the ER to $4 \mathrm{~h}$ after diagnosis of acute pancreatitis. We did not utilize the entire time period in the ER due to important differences in ER length of stay between centers (data not shown). We selected this very early time period to avoid the confounding due to fluid sequestration and reverse causation bias. ${ }^{27}$ FVER was divided into three groups based on tertiles across the study cohort: a) nonaggressive fluid volume group (reference group for comparisons): patients receiving $<500 \mathrm{ml}$ of FVER; b) moderate fluid volume group: between 500 and $1000 \mathrm{ml}$; and c) aggressive fluid volume group: $>1000 \mathrm{ml}$. The total amount of fluid volume administered in the first $24 \mathrm{~h}$ (FV24) was calculated and was also divided into tertiles: a) nonaggressive fluid volume group (reference group for comparisons): patients receiving $<3200 \mathrm{ml}$ of FV24; b) moderate fluid volume group: between 3200 and $4300 \mathrm{ml}$; and c) aggressive fluid volume group: $>4300 \mathrm{ml}$. We hypothesized that the effect of FV24 on outcomes would be more influenced than FVER by fluid sequestration due to the development of local complications, SIRS and organ failure in patients with moderate-to-severe disease.

\section{Outcome variables}

Local complications and persistent organ failure were defined according to the Revised Atlanta Classification. $^{29}$ Local complications included acute peripancreatic fluid collections and/or pancreatic necrosis and/or peripancreatic necrosis. Interventions 


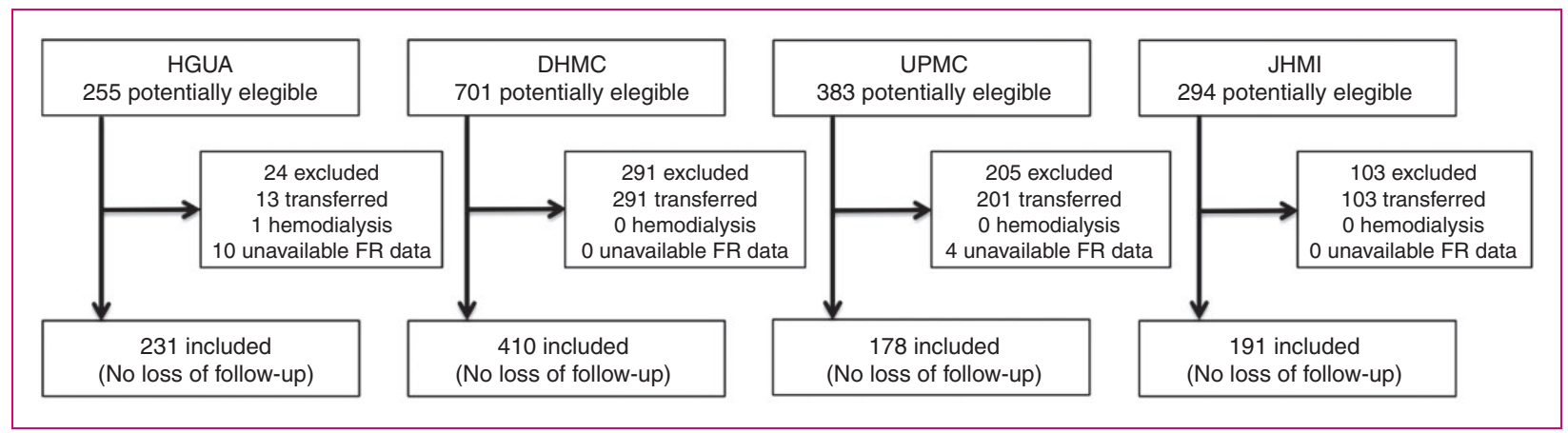

Figure 1. Sources of the study population.

HGUA: Hospital General Universitario de Alicante; DHMC: Dartmouth-Hitchcock Medical Center; UPMC: University of Pittsburgh Medical Center; JHMI: Johns Hopkins Medical Institutions; FR: fluid resuscitation.

were defined as the need for drainage (percutaneous and/or endoscopic) and/or necrosectomy (either endoscopic and/or surgical). Mortality was defined as death occurring during index hospitalization or later during subsequent admissions due to complications of the disease.

\section{Statistical analysis}

The baseline characteristics and outcomes of patients were expressed as median (interquartile range) or mean (standard deviation) for non-normally or normally distributed quantitative data; frequencies and percentages were given for qualitative variables. The Shapiro-Wilk test was used to assess the normality of the distributions. Statistical differences were analyzed using the chisquare test for categorical data and the Mann-Whitney $U$ or Student $t$ test for non-normally or normally distributed quantitative data when comparing two groups, respectively; when more than two groups were compared, Kruskal-Wallis test or one-way analysis of variance were used. Outcomes in the moderate and aggressive FVER and FV24 categories were compared with the patients with nonaggressive resuscitation using chi-square test with Bonferroni correction (two-side level of statistical significance for two post-hoc comparisons: 0.025$)$. All other reported $p$ values are also two-sided and $p$ values $<0.05$ were deemed statistically significant.

For the multivariable analysis (logistic regression), we selected variables obtained at presentation that potentially influence the administration of more or less fluid based on prior studies. These variables included: age $>60$ years, ${ }^{24,26,30}$ alcoholic etiology, ${ }^{24,26,32}$ hematocrit $>44 \%,{ }^{24,26,30}$ blood urea nitrogen $>25 \mathrm{mg} / \mathrm{dl},{ }^{30}$ and presence of SIRS; ${ }^{24,26,30}$ finally, the center of origin was included due to differences in fluid administration and outcomes between the institutions. Results were expressed as odds ratios (ORs) and adjusted ORs with the corresponding 95\% confidence intervals (CIs). Multiple linear regression was used to analyze the adjusted influence of FVER and FV24 on hospital stay.

All statistical analysis was performed using SPSS 19.0 (SPSS, Inc., Chicago, Illinois, USA).

\section{Results}

A total of 1010 patients were included: 231 (22.9\%) patients from HGUA, admitted between August 2010 and November 2013; $410(40.6 \%)$ patients from DHMC, admitted between January 1985 and December $2010 ; 178(17.6 \%)$ patients from UPMC, admitted between June 2003 and August 2013; and $191(18.9 \%)$ patients from JHMI, admitted between January 2010 and March 2013 (Figure 1). Mean time from arrival at the ER to the diagnosis of acute pancreatitis was $3.2 \mathrm{~h}$ (SD: 1.5); there were no differences between centers. Mean FVER was $970 \pm 894 \mathrm{ml}$. The tertiles (p33 and p66) for FVER were 500 and $1000 \mathrm{ml}$. By stratification of the study cohort into tertiles of FVER, $269(26.6 \%)$ patients received $<500 \mathrm{ml}$, $427(42.3 \%)$ received between 500 and $1000 \mathrm{ml}$, and 314 $(31.1 \%)$ received $>1000 \mathrm{ml}$. The histogram of FVER is displayed in Figure 2. Baseline characteristics of the patient cohort stratified by tertiles of FVER are displayed in Table 1 . There were statistically significant differences between the FVER group with regard to age (lower in the aggressive resuscitation group) and SIRS at presentation (more frequent in the aggressive resuscitation group).

The frequency and comparison of outcomes by the tertiles of FVER are displayed in Table 2. Compared with the nonaggressive fluid volume group, the moderate volume group was associated with lower rates of local complications (in the unadjusted analysis) and interventions (both in unadjusted and adjusted analysis). The aggressive resuscitation group was significantly associated with lower need for interventions 
in both the unadjusted and the adjusted analysis. Detailed local complications are displayed in Table 1 in the Supplementary Material online. Compared with the nonaggressive resuscitation group, there was a trend towards a lower rate of acute peripancreatic fluid collections and pancreatic necrosis as well as significant differences regarding peripancreatic necrosis (only in univariable analysis) in the moderate resuscitation group as well as lower rates of peripancreatic necrosis (only in univariable analysis) in the aggressive resuscitation group (Supplementary Table 1 online).

The median (Q1-Q3) length of hospital stay was 7 (4-11), 6 (4-10) and 5 (3-8) days for the nonaggressive, moderate and aggressive FVER volume groups,

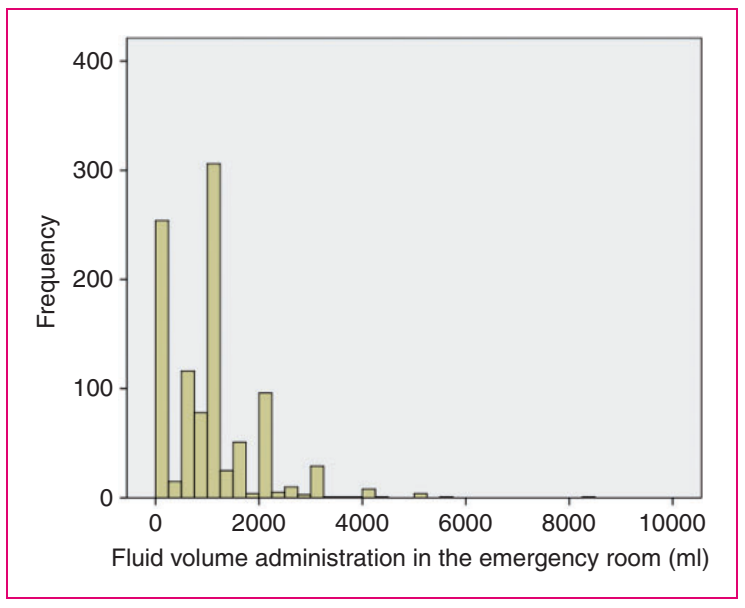

Figure 2. Distribution of fluid volume administration in emergency room. respectively ( $p<0.01$ in univariable but $p>0.05$ in multivariable analysis).

We had data regarding FV24 for 765 (75.7\%) patients. Mean (SD) FV24 was 3909 (1547) ml. The tertiles (p33 and p66) for FV24 were 3200 and $4300 \mathrm{ml}$. The frequency and comparison of outcomes by the tertiles of FV24 are displayed in Table 3. Receiving $>4300 \mathrm{ml}$ of FV24 was associated with local complications on univariable and multivariable analysis and to invasive treatment only in univariable analysis. The median (Q1-Q3) length of hospital stay was 5 (3-8), 6 (4-9) and 6.25 (4-11) days for the nonaggressive, moderate and aggressive FV24 volume groups $(p<0.01$ in univariable but $p>0.05$ in multivariable analysis).

The baseline characteristics by center of origin are displayed in Supplementary Table 2 online. There were significant differences between centers for age, etiology, hematocrit, presence of SIRS and BISAP score. Mean age was higher in HGUA; gallstone-related acute pancreatitis was more frequently found in HGUA and JHMI. Prediction of severity according to a BISAP score $\geq 3$ and hematocrit $\geq 44 \%$ was also more frequent in HGUA. JHMI had a higher proportion of patients with SIRS criteria at the ER. FVER boxplots according to center of origin are displayed in Figure 3. The differences between centers were statistically significant. JHMI and DHMC administered higher FVER than HGUA and UPMC.

The outcomes stratified by center of origin are displayed in Supplementary Table 3. There were statistically significant differences between centers with regard to the incidence of local complications (higher rate in HGUA), interventions (more frequent in HGUA and

Table 1. Baseline characteristics according to fluid volume administration in the emergency room.

\begin{tabular}{|c|c|c|c|c|c|}
\hline \multirow[b]{2}{*}{ Variable } & \multirow[b]{2}{*}{$\begin{array}{l}\text { Total cohort } \\
(N=1010)\end{array}$} & \multicolumn{3}{|l|}{ FVER } & \multirow[b]{2}{*}{$p$} \\
\hline & & $\begin{array}{l}<500 \mathrm{ml} \\
(n=269)\end{array}$ & $\begin{array}{l}500-1000 \mathrm{ml} \\
(n=427)\end{array}$ & $\begin{array}{l}>1000 \mathrm{ml} \\
(n=314)\end{array}$ & \\
\hline Age, years & $53.6(19.6)$ & $55.4(18.6)$ & $54.3(20.3)$ & $49.5(17.3)$ & $<0.001$ \\
\hline Male & $508(50.3 \%)$ & $125(46.5 \%)$ & $215(50.4 \%)$ & $168(53.5 \%)$ & 0.238 \\
\hline \multicolumn{6}{|l|}{ Etiology } \\
\hline Biliary & 448 (44.4\%) & $114(42.4 \%)$ & $196(45.9 \%)$ & $138(43.9 \%)$ & 0.139 \\
\hline Alcohol & $139(13.8 \%)$ & $36(13.4 \%)$ & $54(12.6 \%)$ & $49(15.6 \%)$ & \\
\hline Other & $423(41.9 \%)$ & $119(44.2 \%)$ & $177(41.5 \%)$ & 127 (40.4\%) & \\
\hline $\mathrm{BISAP} \geq 3$ & $47(4.7 \%)$ & $17(6.3 \%)$ & $17(4.1 \%)$ & $13(4.3 \%)$ & 0.375 \\
\hline Hematocrit $\geq 44 \%$ & $285(28.2 \%)$ & $73(27.1 \%)$ & $123(28.8 \%)$ & $89(28.3 \%)$ & 0.757 \\
\hline $\mathrm{BUN} \geq 25 \mathrm{mg} / \mathrm{dl}$ & $132(13.1 \%)$ & $33(12.3 \%)$ & $52(12.2)$ & $47(15 \%)$ & 0.433 \\
\hline SIRS & 347 (34.4\%) & $89(33.1 \%)$ & $130(30.4 \%)$ & 128 (40.8\%) & 0.012 \\
\hline
\end{tabular}

Data expressed as mean (SD) or number (\%).

FVER: fluid volume administration in the emergency room; BISAP: bedside index for severity of acute pancreatitis; BUN: blood urea nitrogen; SIRS: systemic inflammatory response syndrome 
Table 2. Frequency and comparison of outcomes stratified by tertiles of fluid volume administration in the emergency room.

\begin{tabular}{|c|c|c|c|c|}
\hline FVER & $\begin{array}{l}\text { Local } \\
\text { complications }\end{array}$ & $\begin{array}{l}\text { Persistent organ } \\
\text { failure }\end{array}$ & $\begin{array}{l}\text { Invasive } \\
\text { treatment }\end{array}$ & Death \\
\hline \multicolumn{5}{|l|}{$<500 \mathrm{ml}$} \\
\hline Cases & $51 / 269$ (19\%) & $19 / 269(7.1 \%)$ & $13 / 269(4.8 \%)$ & $8 / 269(3 \%)$ \\
\hline OR $(95 \% \mathrm{Cl})$ & 1 & 1 & 1 & 1 \\
\hline ORa $(95 \% \mathrm{Cl})$ & 1 & 1 & 1 & 1 \\
\hline \multicolumn{5}{|l|}{$500-1000 \mathrm{ml}$} \\
\hline Cases & $48 / 427(11.2 \%)$ & $19 / 427(4.4 \%)$ & $7 / 427$ (1.6\%) & $7 / 427$ (1.6\%) \\
\hline OR $(95 \% \mathrm{Cl})$ & $0.54(0.35-0.83)^{a}$ & $0.61(0.32-1.18)$ & $0.33(0.13-0.83)^{a}$ & $0.54(0.2-1.52)$ \\
\hline ORa $(95 \% \mathrm{Cl})$ & $0.67(0.43-1.05)$ & $0.56(0.28-1.14)$ & $0.37(0.14-0.98)^{b}$ & $0.46(0.15-1.38)$ \\
\hline \multicolumn{5}{|l|}{$>1000 \mathrm{ml}$} \\
\hline Cases & $50 / 314(15.9 \%)$ & $15 / 314(4.8 \%)$ & $5 / 314(1.6 \%)$ & $8 / 314(2.5 \%)$ \\
\hline OR (95\% Cl) & $0.81(0.53-1.24)$ & $0.66(0.33-1.33)$ & $0.32(0.11-0.91)^{b}$ & $0.85(0.32-2.3)$ \\
\hline ORa $(95 \% \mathrm{CI})$ & $1.15(0.71-1.86)$ & $0.5(0.22-1.12)$ & $0.21(0.05-0.84)^{a}$ & $0.64(0.20-2)$ \\
\hline
\end{tabular}

Reference group for comparisons: FVER $<500 \mathrm{ml}$. Cases: expressed as number of cases with outcome variable/number of patients within the category of fluid administration (percent cases within category of fluid administration).

Variables included in the multivariate analysis: age $>60$, alcoholic etiology, hematocrit $>44 \%$, blood urea nitrogen $>25 \mathrm{mg} / \mathrm{dl}$, presence of systemic inflammatory response syndrome and center of origin.

${ }^{a} p<0.025$ vs. reference group.

${ }^{\mathrm{b}} p=0.03$ vs. reference group.

FVER: fluid volume administration in the emergency room; OR: odds ratio; ORa: adjusted OR; CI: confidence interval

Table 3. Frequency and comparison of outcomes stratified by tertiles of fluid volume administration in the first $24 \mathrm{~h}$.

\begin{tabular}{|c|c|c|c|c|}
\hline FV24 & $\begin{array}{l}\text { Local } \\
\text { complications }\end{array}$ & $\begin{array}{l}\text { Persistent } \\
\text { organ failure }\end{array}$ & $\begin{array}{l}\text { Invasive } \\
\text { treatment }\end{array}$ & Death \\
\hline \multicolumn{5}{|l|}{$<3200 \mathrm{ml}$} \\
\hline Cases & $30 / 260$ (11.5\%) & $11 / 260(4.2 \%)$ & $2 / 260(0.8 \%)$ & $4 / 260(1.5 \%)$ \\
\hline OR (95\% Cl) & 1 & 1 & 1 & 1 \\
\hline$O R a(95 \% \mathrm{Cl})$ & 1 & 1 & 1 & 1 \\
\hline \multicolumn{5}{|l|}{$3200-4300 \mathrm{ml}$} \\
\hline Cases & 41/249 (16.5\%) & $9 / 249$ (3.6\%) & $6 / 249(2.4 \%)$ & $3 / 249(1.2 \%)$ \\
\hline OR $(95 \% \mathrm{Cl})$ & $1.51(0.91-2.51)$ & $0.85(0.35-2.09)$ & $3.19(0.64-15.93)$ & $0.78(0.17-3.52)$ \\
\hline ORa $(95 \% \mathrm{Cl})$ & $1.28(0.76-2.16)$ & $0.85(0.34-2.15)$ & $2.62(0.52-13.36)$ & $0.83(0.18-3.92)$ \\
\hline \multicolumn{5}{|l|}{$>4300 \mathrm{ml}$} \\
\hline Cases & $65 / 256(25.4 \%)$ & $16 / 256(6.2 \%)$ & $13 / 256(5.1 \%)$ & $5 / 256(2 \%)$ \\
\hline OR $(95 \% \mathrm{Cl})$ & $2.61(1.63-4.19)^{a}$ & $1.51(0.69-3.32)$ & $6.9(1.54-30.9)^{\mathrm{a}}$ & $1.28(0.338-4.8)$ \\
\hline ORa $(95 \% \mathrm{CI})$ & $1.91(1.17-3.14)^{\mathrm{a}}$ & $1.57(0.67-3.72)$ & $4.5(0.99-21.07)$ & $1.78(0.43-7.46)$ \\
\hline
\end{tabular}

Reference group for comparisons: FV24 < 3200 ml. Cases: expressed as number of cases with outcome variable/number of patients within the category of fluid administration (percent cases within category of fluid administration).

Variables included in the multivariate analysis: age $>60$, alcoholic etiology, hematocrit $>44 \%$, blood urea nitrogen $>25 \mathrm{mg} / \mathrm{dl}$, presence of systemic inflammatory response syndrome and center of origin.

${ }^{a} p<0.025$ vs. reference group.

FV24: fluid volume administration in the first $24 \mathrm{~h}$; OR: odds ratio; ORa: adjusted OR; Cl: confidence interval 


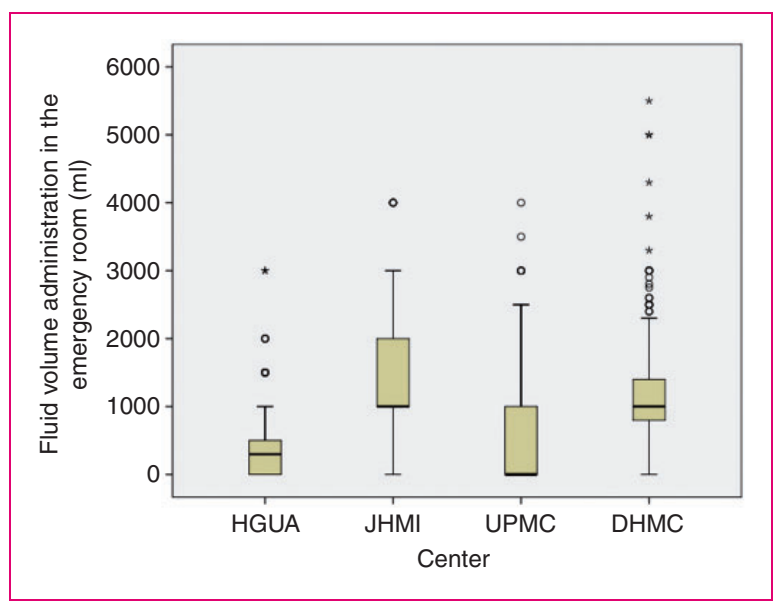

Figure 3. Fluid volume administration in the emergency room according to center of origin. Centers: Hospital General Universitario de Alicante (HGUA), Johns Hopkins Medical Institutions (JHMI), University of Pittsburgh Medical Center (UPMC), Dartmouth-Hitchcock Medical Center (DHMC). The differences in emergency room volume administration between centers were statistically significant $(p<0.05)$.

UPMC), and length of hospital stay (longer in HGUA); see Supplementary Table 3.

\section{Discussion}

The present multicenter study is the largest to date evaluating the outcomes of early fluid administration in patients with acute pancreatitis. As there is no specific threshold to define aggressive fluid resuscitation, we pragmatically divided our patients according to tertiles. Three groups give more information than two that include extreme cases of fluid administration. If we choose a dichotomous variable, nonaggressive versus aggressive fluid administration, we are comparing a group which includes some patients with extremely low administration of fluids in the ER with another group in which some patients are receiving extremely high volumes, and this fact may distort the real relationship between volume and outcome. We performed a multivariate analysis with those variables that may influence the attending physician to administer a higher volume of fluid resuscitation, before the patients have "declared" severity of disease and fluid sequestration. A moderate volume of fluid $(500-1000 \mathrm{ml})$ administered in the ER is associated with a lower incidence of local complications compared with the nonaggressive $(<500 \mathrm{ml})$ group in univariable analysis, but it did not remain significant after adjusting for potential confounders. This may be due to the relatively low number of events (with an overall 14.8\% local complication frequency) adjusted by a regression model with a high number of variables. This lower number of events compared with other studies is due to the exclusion of transferred patients to avoid biases regarding previous fluid resuscitation. The purpose of this study was to investigate the real effect of early fluid resuscitation on outcomes, so we carefully selected each variable that may be a confounder.

The clinical evidence supporting the type, rate, and amount of fluid administered to patients with acute pancreatitis is limited. Two controlled trials from China (from the same center) found that aggressive fluid resuscitation is associated with worse outcomes. ${ }^{22,23}$ In a recent systematic review ${ }^{33}$ these two studies were rated as moderate with regard to level of evidence as they were not blinded and, in one of the trials, a pseudorandomized allocation was used. ${ }^{23}$ The amount of crystalloid and colloid fluid administered was large in both arms of the first study using weight-based fluid administration for rapid fluid expansion versus controlled fluid expansion. $^{22}$ The rapid expansion group had a higher need for mechanical ventilation, and higher sepsis and mortality. In their second study, fluid resuscitation was directed using hematocrit to rapidly or gradually reverse hemoconcentration. ${ }^{23}$ The rapid hemodilution group had a higher incidence of sepsis and increased in-hospital mortality. While these data are not directly comparable with ours due to their inclusion of only patients with severe acute pancreatitis, they suggest that over-resuscitation may be detrimental. In the first study by Mao et al. ${ }^{22}$, patients with severe acute pancreatitis were enrolled in the first $72 \mathrm{~h}$ after the onset of severe disease. Local complications are known to be established after three to four days from the onset of acute pancreatitis, ${ }^{34}$ so that study in fact does not intend to decrease the rate of severe acute pancreatitis by means of fluid resuscitation; to the contrary it guides the management of fluid therapy in established severe acute pancreatitis in an intensive care unit environment. In the second study ${ }^{23}$ patients were enrolled within the first $24 \mathrm{~h}$ after the onset of disease but, again, the study focused on established severe acute pancreatitis.

In our study, early aggressive fluid resuscitation was not associated with decreased rates of persistent organ failure, local complications nor death, so the importance of this supportive measure may not be as important as stated. Aggressive resuscitation in the first $24 \mathrm{~h}$ was associated with local complications, presumably due to reverse causation bias (higher requirements of fluid volume due to increased fluid sequestration induced by retroperitoneal accumulation of liquid). Furthermore we described a trend towards a lower hospital stay in patients receiving more aggressive resuscitation in the ER versus a trend towards a higher hospital stay in patients receiving more fluids in the first $24 \mathrm{~h}$. These findings support our theory about reverse causation bias in observational studies 
addressing fluid resuscitation in acute pancreatitis. Alternatively we can hypothesize that fluid overload may be detrimental if maintained beyond an early and short-lasting therapeutic window. Our study suggests, with the limitations of a retrospective study, that we have to focus on very early fluid resuscitation to demonstrate the clinical benefit of moderate to aggressive volume administration.

A randomized controlled trial (RCT) from the USA by $\mathrm{Wu}$ et al. ${ }^{35}$ compared conventional with goal-directed fluid resuscitation (mainly based on changes in blood urea nitrogen) and failed to demonstrate any differences between the two groups. ${ }^{35}$ This study randomized only 40 patients, and the volume of fluid administered to both groups of patients was similar. A recent RCT by Buxbaum et al. $^{36}$ reported a benefit of aggressive versus standard fluid administration for preventing post-endoscopic retrograde cholangiopancreatography pancreatitis $^{36}$ but they studied a different setting: the prevention of acute pancreatitis. Observational studies both in favor of ${ }^{9,10,15,37}$ and against ${ }^{20,24}$ aggressive fluid administration are biased by reverse causation and/or their indirect nature, as commented on before.

There appear to be two clinical phases that determine the volume of fluid administration in acute pancreatitis: ${ }^{27}$ an early phase determined by the clinician and a late phase determined by the specific patient response to fluid therapy. In the early phase (ER and the initial hours after hospital admission), fluid volume administration is determined by the clinician based on a complex heuristic model that probably includes a number of initial clinical signs and laboratory results as well as personal experience and general management strategy (aggressive versus nonaggressive fluid administration). However, in the late phase, fluid volume administration mainly depends on the patient's response to initial therapy as patients with fluid sequestration (which includes primarily those with local complications, SIRS, and organ failure) may need an increase in fluid volume administration to maintain urine output and blood pressure and to normalize laboratory markers of hypovolemia, including hematocrit, blood urea nitrogen, and creatinine. ${ }^{26}$ Conversely, some patients will have a rapid recovery and may develop polyuria, so the fluid administration rate may be decreased or stopped altogether. This late phase makes it difficult to study the effect of fluid volume administration on outcomes due to reverse causation bias. ${ }^{27}$ Furthermore, in this late phase, local and systemic complications may be well-established and cannot be prevented by aggressive fluid volume administration.

This study has several strengths. Our design attempts to adjust for the confounding that occurs due to variable timing, reverse causation bias, and lack of adjustment for laboratory and clinical variables that influence clinician decision making with regard to fluid management. Our large sample size, comprising patients from several centers, presumably increases the external validity of our results. The exclusion of transferred patients is particularly important as they often have severe acute pancreatitis and have received fluid resuscitation in their primary care centers.

The primary limitation of our study is its retrospective nature. Despite the fact that there were differences between the centers with regard to baseline characteristics, fluid administration, and outcomes, we adjusted for the center of origin in our multivariable analysis. We were not able to adjust for the type of fluid administered in the centers; therefore, the use of lactated Ringer's after 2011 at one and not another center could have affected rates of SIRS. However, since we examined only the first several hours of presentation in the ER, it is unlikely that lactated Ringer's would lead to resolution of SIRS so rapidly and we adjusted for the presence of SIRS in the association between FVER and outcome. The patients enrolled were from different time periods in the different study centers, which may lead to biases. The low number of events, due to the exclusion of transferred patients, may lead to a decreased power to detect differences, as commented before.

More definitive evidence regarding the effects of early fluid administration on outcomes of acute pancreatitis should be ascertained by means of a RCT.

In conclusion, our study suggests that early moderate to aggressive fluid volume administration in the ER is associated with significantly less need for invasive interventions in patients with acute pancreatitis.

\section{Declaration of conflicting interests}

The authors declare that there is no conflict of interest.

\section{Funding}

This research received no specific grant from any funding agency in the public, commercial, or not-for-profit sectors.

\section{References}

1. Peery AF, Crockett SD, Barritt AS, et al. Burden of gastrointestinal, liver, and pancreatic diseases in the United States. Gastroenterology 2015; 149: 1731-1741 e3.

2. Dervenis C, Johnson CD, Bassi C, et al. Diagnosis, objective assessment of severity, and management of acute pancreatitis. Santorini consensus conference. Int J Pancreatol 1999; 25: 195-210.

3. Banks PA and Freeman ML; Practice Parameters Committee of the American College of G. Practice guidelines in acute pancreatitis. Am J Gastroenterol 2006; 101: 2379-2400.

4. Whitcomb DC. Clinical practice. Acute pancreatitis. $N$ Engl J Med 2006; 354: 2142-2150. 
5. Pandol SJ, Saluja AK, Imrie CW, et al. Acute pancreatitis: bench to the bedside. Gastroenterology 2007; 132: 1127-1151.

6. Navarro S, Amador J, Arguello L, et al. [Recommendations of the Spanish Biliopancreatic Club for the Treatment of Acute Pancreatitis. Consensus development conference]. Gastroenterol Hepatol 2008; 31: 366-387.

7. Tenner S, Baillie J, DeWitt J, et al.; American College of Gastroenterology. American College of Gastroenterology guideline: Management of acute pancreatitis. Am J Gastroenterol 2013; 108: 1400-1415; 16.

8. Gardner TB, Vege SS, Pearson RK, et al. Fluid resuscitation in acute pancreatitis. Clin Gastroenterol Hepatol 2008; 6: 1070-1076.

9. Gardner TB, Vege SS, Chari ST, et al. Faster rate of initial fluid resuscitation in severe acute pancreatitis diminishes in-hospital mortality. Pancreatology 2009; 9: $770-776$.

10. Warndorf MG, Kurtzman JT, Bartel MJ, et al. Early fluid resuscitation reduces morbidity among patients with acute pancreatitis. Clin Gastroenterol Hepatol 2011; 9: 705-709.

11. Baillargeon JD, Orav J, Ramagopal V, et al. Hemoconcentration as an early risk factor for necrotizing pancreatitis. Am J Gastroenterol 1998; 93: 2130-2134.

12. Brown A, Orav $\mathbf{J}$ and Banks PA. Hemoconcentration is an early marker for organ failure and necrotizing pancreatitis. Pancreas 2000; 20: 367-372.

13. Gan SI and Romagnuolo J. Admission hematocrit: A simple, useful and early predictor of severe pancreatitis. Dig Dis Sci 2004; 49: 1946-1952.

14. Wu BU, Bakker OJ, Papachristou GI, et al. Blood urea nitrogen in the early assessment of acute pancreatitis: An international validation study. Arch Intern Med 2011; 171: 669-676.

15. Wall I, Badalov N, Baradarian R, et al. Decreased mortality in acute pancreatitis related to early aggressive hydration. Pancreas 2011; 40: 547-550.

16. Tenner S. Initial management of acute pancreatitis: Critical issues during the first 72 hours. Am J Gastroenterol 2004; 99: 2489-2494.

17. Baron TH and Morgan DE. Acute necrotizing pancreatitis. N Engl J Med 1999; 340: 1412-1417.

18. Clancy TE and Ashley SW. Current management of necrotizing pancreatitis. Adv Surg 2002; 36: 103-121.

19. Whitcomb DC, Muddana V, Langmead CJ, et al. Angiopoietin-2, a regulator of vascular permeability in inflammation, is associated with persistent organ failure in patients with acute pancreatitis from the United States and Germany. Am J Gastroenterol 2010; 105: 2287-2292.

20. Eckerwall G, Olin H, Andersson B, et al. Fluid resuscitation and nutritional support during severe acute pancreatitis in the past: What have we learned and how can we do better? Clin Nutr 2006; 25: 497-504.

21. Weitz G, Woitalla J, Wellhoner P, et al. Detrimental effect of high volume fluid administration in acute pancreatitis - a retrospective analysis of 391 patients. Pancreatology 2014; 14: 478-483.

22. Mao EQ, Tang YQ, Fei J, et al. Fluid therapy for severe acute pancreatitis in acute response stage. Chinese Med J (Engl) 2009; 122: 169-173.

23. Mao EQ, Fei J, Peng YB, et al. Rapid hemodilution is associated with increased sepsis and mortality among patients with severe acute pancreatitis. Chinese Med $J$ (Engl) 2010; 123: 1639-1644.

24. de-Madaria E, Soler-Sala G, Sanchez-Paya J, et al. Influence of fluid therapy on the prognosis of acute pancreatitis: A prospective cohort study. Am J Gastroenterol 2011; 106: 1843-1850.

25. Rothman KJ, Greenland S and Lash TL. Modern epidemiology, 3rd ed. Philadelphia: Lippincott, Williams \& Wilkins, 2008.

26. de-Madaria E, Banks PA, Moya-Hoyo N, et al. Early factors associated with fluid sequestration and outcomes of patients with acute pancreatitis. Clin Gastroenterol Hepatol 2014; 12: 997-1002.

27. de-Madaria E, Martinez J and Perez-Mateo M. The dynamic nature of fluid resuscitation in acute pancreatitis. Clin Gastroenterol Hepatol 2012; 10: 95-96 author reply 6.

28. Norman J. The role of cytokines in the pathogenesis of acute pancreatitis. Am J Surg 1998; 175: 76-83.

29. Banks PA, Bollen TL, Dervenis C, et al. Classification of acute pancreatitis - 2012: Revision of the Atlanta classification and definitions by international consensus. Gut 2013; 62: 102-111.

30. Wu BU, Johannes RS, Sun X, et al. The early prediction of mortality in acute pancreatitis: A large populationbased study. Gut 2008; 57: 1698-1703.

31. Papachristou GI, Muddana V, Yadav D, et al. Comparison of BISAP, Ranson's, APACHE-II, and CTSI scores in predicting organ failure, complications, and mortality in acute pancreatitis. Am J Gastroenterol 2010; 105: 435-441.

32. Papachristou GI, Papachristou DJ, Morinville VD, et al. Chronic alcohol consumption is a major risk factor for pancreatic necrosis in acute pancreatitis. Am J Gastroenterol 2006; 101: 2605-2610.

33. Haydock MD, Mittal A, Wilms HR, et al. Fluid therapy in acute pancreatitis: Anybody's guess. Ann Surg 2013; 257: $182-188$.

34. Isenmann R, Buchler M, Uhl W, et al. Pancreatic necrosis: An early finding in severe acute pancreatitis. Pancreas 1993; 8: 358-361.

35. $\mathrm{Wu}$ BU, Hwang JQ, Gardner TH, et al. Lactated Ringer's solution reduces systemic inflammation compared with saline in patients with acute pancreatitis. Clin Gastroenterol Hepatol 2011; 9: 710-717 e1.

36. Buxbaum J, Yan A, Yeh K, et al. Aggressive hydration with lactated Ringer's solution reduces pancreatitis after endoscopic retrograde cholangiopancreatography. Clin Gastroenterol Hepatol 2014; 12: 303-307 e1.

37. Brown A, Baillargeon JD, Hughes MD, et al. Can fluid resuscitation prevent pancreatic necrosis in severe acute pancreatitis? Pancreatology 2002; 2: 104-107. 